\title{
Differentiation and management of hepatobiliary mucinous cystic neoplasms: a single centre experience for 8 years
}

Jiaqi Gao, Junhao Zheng, Jingwei Cai, Mubarak Ali Kirih, Junjie Xu, Liye Tao, Yuelong Liang, Xu Feng, Jing Fang and Xiao Liang* ${ }^{*}$

\begin{abstract}
Background: Hepatobiliary mucinous cystic neoplasms (H-MCNs) are relatively rare cystic neoplasms in the liver. The differential diagnosis of H-MCNs remains big challenging, and the management and prognosis between the hepatic simple cyst (HSC) and H-MCNs are quite different. This study aimed to present our experience in the management of $\mathrm{H}-\mathrm{MCN}$ s and provide a preoperative H-MCNs risk prediction nomogram to differentiating H-MCNs from liver cystic lesions.
\end{abstract}

Methods: 29 patients diagnosed with H-MCNs and 75 patients diagnosed with HSC between June 2011 and June 2019 at Zhejiang University School of medicine, Sir Run-Run Shaw Hospital were reviewed in this study. We analyzed the demographic and clinicopathological variables.

Results: US, CT, and MRI could accurately diagnose only $3.4 \%, 46.1 \%$, and $57.1 \%$ of H-MCNs, respectively. After univariate analysis and multivariate logistic regression analysis, the variables significantly associated with H-MCNs were enhancement after contrast $(p=0.009)$, tumour located in the left lobe $(p=0.02)$ and biliary ductal dilation $(p=0.027$ ). An H-MCNs risk predictive nomogram was constructed, which showed excellent discrimination (areas under the receiver operating characteristic curve were 0.940) and consistent calibration between the predicted probability and actual probability.

Conclusion: Among patients with H-MCNs, the location of the tumour, enhancement in CT scan, and biliary duct dilation are significantly independent risk factors. The appropriate treatment of H-MCNs is radical resection. Using our Nomogram could facilitate screening and identification of patients with liver cystic lesions.

Keywords: Cystadenoma, Liver, Diagnosis, Surgical procedures

\section{Background}

With the development of the radiography technique and the increasing frequency of routine health examination, more and more liver cystic lesions have been discovered throughout these years. It was reported that about $20 \%$

\footnotetext{
*Correspondence: srrshlx@zju.edu.cn
}

Department of General Surgery, School of Medicine, Sir Run Run Shaw Hospital, Zhejiang University, 3 East Qingchun Road, Hangzhou 310016, Zhejiang, China of ordinary people have liver cystic lesions [1]. The most majority of cystic lesions are hepatic simple cysts (HSC), a kind of common benign cysts of the liver. HSC grows up by inches, and most of the HSC require no treatment or just fenestration [2]. However, there are other two more aggressive lesions in the liver, called intrahepatic biliary cystadenomas (IBC) and intrahepatic biliary cystadenocarcinoma (IBAC). IBC and IBAC are estimated to occupy less than $5 \%$ of the liver cysts $[3,4]$. IBC was first reported by Henter et al. in 1887 [5]. Then in 1958, 
Emre described IBC as a liver cystic neoplasm with the pathological feature of "ovarian-like stroma(OS)."[6] By the year of 2010, the World Health Organization (WHO) redefined that the $\mathrm{OS}$ is the requirement for diagnosing cystadenoma both in liver and pancreas, and rename the IBC and IBAC as hepatobiliary mucinous cystic neoplasms (H-MCNs) $[7,8]$.

Although the equipment of imaging diagnosis has been improving quite a lot, such as contrasted-enhanced ultrasound (US), computed tomography (CT) and magnetic resonance imaging (MRI), the differential diagnosis for $\mathrm{H}-\mathrm{MCNs}$ and HSC was still a big challenge [9]. IBC has a malignant potential to transform into IBAC in $20-30 \%$ patients, and the recurrent rate is much higher than HSC after fenestration therapy [10]. Because the biological characteristics and treatment strategies between $\mathrm{H}-\mathrm{MCNs}$ and HSC are quite different, the accurate preoperative diagnosis of $\mathrm{H}-\mathrm{MCNs}$ is of great significance. This study aimed to present our experience in the diagnosis and management of $\mathrm{H}-\mathrm{MCNs}$ under the new WHO classification. Meanwhile, we developed and validated a predictive model to enhance the diagnostic accuracy between $\mathrm{H}-\mathrm{MCNs}$ and $\mathrm{HSC}$, and display the model with a nomogram.

\section{Methods}

From June 2011 to June 2019, a total of 29 patients underwent surgical treatment and pathologically diagnosed as $\mathrm{H}-\mathrm{MCNs}$ within the WHO 2010 new classification standard at Zhejiang University, School of medicine, Sir Run-Run Shaw Hospital. All of the patients in this study underwent a routine pathological examination of surgical specimens. The pathological diagnosis was concluded by at least two experienced pathologists. Besides, 75 patients pathologically diagnosed as HSC between June 2011 and June 2019 were randomly included to compare the characteristics with the H-MCNs. The patients with other liver neoplasms, missing or incomplete data, nonoperative treatment, or these who weren't diagnosed in histology were excluded from the study (Fig. 1). Patients with liver cystic lesions in this cohort were routinely recommended to undergo the US or CT examination. For patients with unclear diagnosis in US or CT were recommended to underwent MRI examination.

Clinical and demographic variables, including age, gender, clinical symptoms, duration of symptoms, serum tumour markers, liver function, were obtained from medical records. Information about the preoperative imaging findings of US, CT, and MRI was reviewed. Radiological features like enhancement after contrast, multilocular cyst, septa, calcification, biliary ductal dilatation were collected. The surgical approaches, operation time, and bleed loss were reviewed from operation records. Tumour size was obtained from the pathological reports, which is defined as the longest diameter of the tumour. If the size was not mentioned in pathological reports, we referred to the most recently preoperative cross-sectional imaging report. The presence of OS was the requirement to diagnosis the $\mathrm{H}-\mathrm{MCNs}$ in histology. This retrospective study was approved by the Ethics committee of Sir Run Run Shaw Hospital (Reference number SRR20200210).

Continuous variables were displayed as the mean \pm standard deviation or medians with ranges. Categorical variables were presented as numbers and percentages. We used student's t-test or Mann-Whitney $\mathrm{U}$ test to compare continuous variables, and chi-square

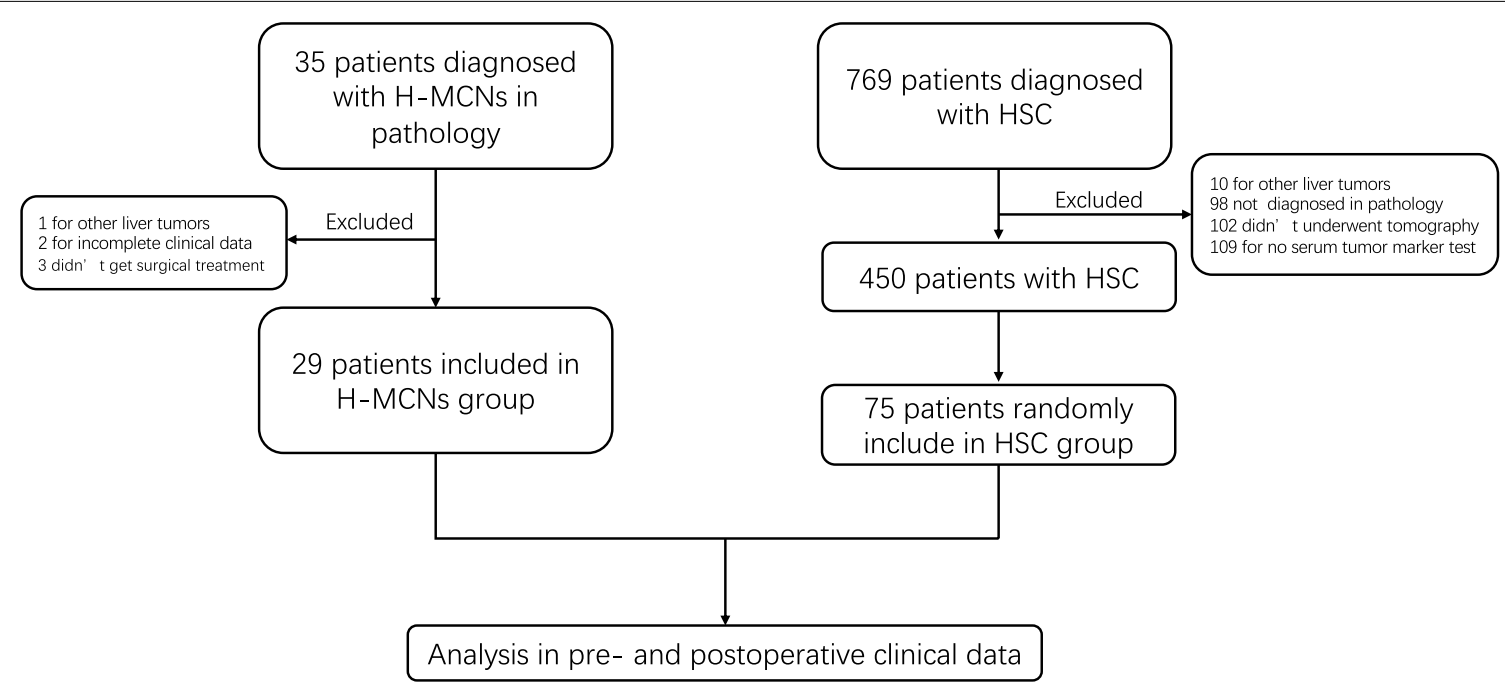

Fig. 1 Flowchart of patient selection 
or Fisher's exact test to compare categorical variables among different groups. The subgroup was set by the cutoff value according to the receiver operating characteristic curve analysis reported by Wang et al.[11]

The univariate analysis was used to analyze the relationship of preoperative clinical characteristics and imaging features between HSC and H-MCN. Variables with statistical significance were further enrolled in the multivariate logistic analysis. We incorporated the variables significantly independent in multivariate logistic analysis to construct a preoperative $\mathrm{H}-\mathrm{MCNs}$ risk prediction nomogram. And we forced factors that have great clinical relevance back into the model. Bootstraps with 1000 resamples were used to validate the calibration of the Nomogram. And we used receiver operating characteristic curve analysis and concordance Index (C-index) to assess the model discrimination. A P-value of less than 0.05 was deemed as statistical significance. All of the statistical analysis was conducted by SPSS (ver 23.0, USA) and R software (ver 3.5.3, USA).

\section{Result}

From June 2011 to December 2018, 29 patients diagnosed as $\mathrm{H}-\mathrm{MCNs}$ and underwent surgical treatment in our institution, including 4 cases of IBAC. The images of US, CT, MRI, and corresponding histology were displayed in Fig. 2. Their demographics and clinical characteristics were presented in Additional file 1. The median age of the patients was 53 years (range, 47-62). A majority of patients were female (69\%). About half of the patients initially complained about abdominal pain (48.3\%); the median duration of symptoms was 48 months (range, 0.3-240 months). Interestingly, although the left part of the liver is much smaller than the right, most of the lesions $(79.3 \%)$ were located on the left lobe.

Preoperative image examination and treatment characteristics between $\mathrm{H}-\mathrm{MCNs}$ and $\mathrm{HSC}$ were shown in Table 1. Although most of the patients with $\mathrm{H}-\mathrm{MCNs}$ underwent US, only 1 (3.4\%) patient was accurately diagnosed by the US. Whereas, among the patients with HSC, the accuracy rate of the US was $90.4 \%$. In the $\mathrm{H}$-MCNs group, 26 and 14 patients respectively underwent CT and MRI, but only 12 (46.1\%), and 8 (57.1\%) of the patients were accurately diagnosed as $\mathrm{H}-\mathrm{MCNs}$ by CT and MRI. The diagnostic accuracy of CT and MR was much higher in the patients with HSC. In our institution, half $(50 \%)$ of the $\mathrm{H}-\mathrm{MCNs}$ were performed a laparoscopic operation, and all (100\%) of the patients with HSC underwent laparoscopic surgery. About the type of surgery, the operative intervention included cyst resection (34.5\%) and partial liver resection (65.5\%); 3 patients were initially performed fenestration, then concerted to radical operation because intraoperative frozen section examination suggested that the lesion may be H-MCNs. Compared with the H-MCNs, most of the patients with HSC (96\%) underwent fenestration; 3 patients $(4 \%)$ were performed radical resection due to misdiagnosing as $\mathrm{H}-\mathrm{MCNs}$. A total of 4 patients (14.2\%) with H-MCNs and one patient with HCS suffered a postoperative complication.

During the study period, a total of 104 patients who had liver cyst lesions and underwent surgical treatment were included. Preoperative demographics and clinical characteristics of $\mathrm{H}-\mathrm{MCNs}$ and HSC were displayed in Table 2. Twenty-three variables were used to be tested as potential predictors of $\mathrm{H}-\mathrm{MCNs}$ in liver cystic lesions. In univariate analysis, eight preoperative variables including abdominal pain $(p=0.024)$, fever $(p=0.005)$, duration of the symptom $(p<0.001)$, serum CA19-9 elevation $(p=0.001)$, enhancement after contrast $(p<0.001)$, biliary ductal dilation $(p<0.001)$, septa $(p=0.002)$, location of the cyst $(\mathrm{p}<0.001)$ were significantly different between the group of $\mathrm{H}-\mathrm{MCNs}$ and HSC. These statistically significant factors were included in further analysis by a multivariable logistic regression model. On multivariable analysis (Table 3), with the reported as odds ratio $(95 \% \mathrm{CI})$, enhancement after contrast (12.1[2.11-100]), lesion located in the left of the liver (11.0[1.71-121]), biliary ductal dilatation (9.80[1.46-92.2]) were independently associated H-MCNs. Although serum CA19-9 level was increased in many patients with $\mathrm{H}-\mathrm{MCNs}$, but the difference was not statistically significant (5.21[1.02-31.9], $\mathrm{p}=0.053)$ in multivariable logistic regression analysis. In consideration of improving the sensitivity of the model, we also included the CA19-9 as a significant factor.

Then, we conducted collinearity diagnosis for these independent risk factors, the variance inflation factor was $1.11,1.03,1.20$, and 1.06 , respectively, which means that there is no collinearity among these four factors, and the model composed of these four variables would be robust.

Based on the result of multivariate logistic regression analysis, these four individual predictive factors were incorporated in a preoperative prediction model. We display the model as a preoperative $\mathrm{H}-\mathrm{MCNs}$ risk prediction nomogram (Fig. 3).

To validate the discrimination of the prediction model, we created a ROC curve of the Nomogram (Fig. 4a), and displayed a $\mathrm{C}$-index of 0.940 , suggesting that the prediction has excellent discrimination. The calibration validation of the model was conducted by the calibration plot by bootstrapping with 1000 resamples (Fig. 4b). The predicted probability of the Nomogram was good agreement with the actual probability. 

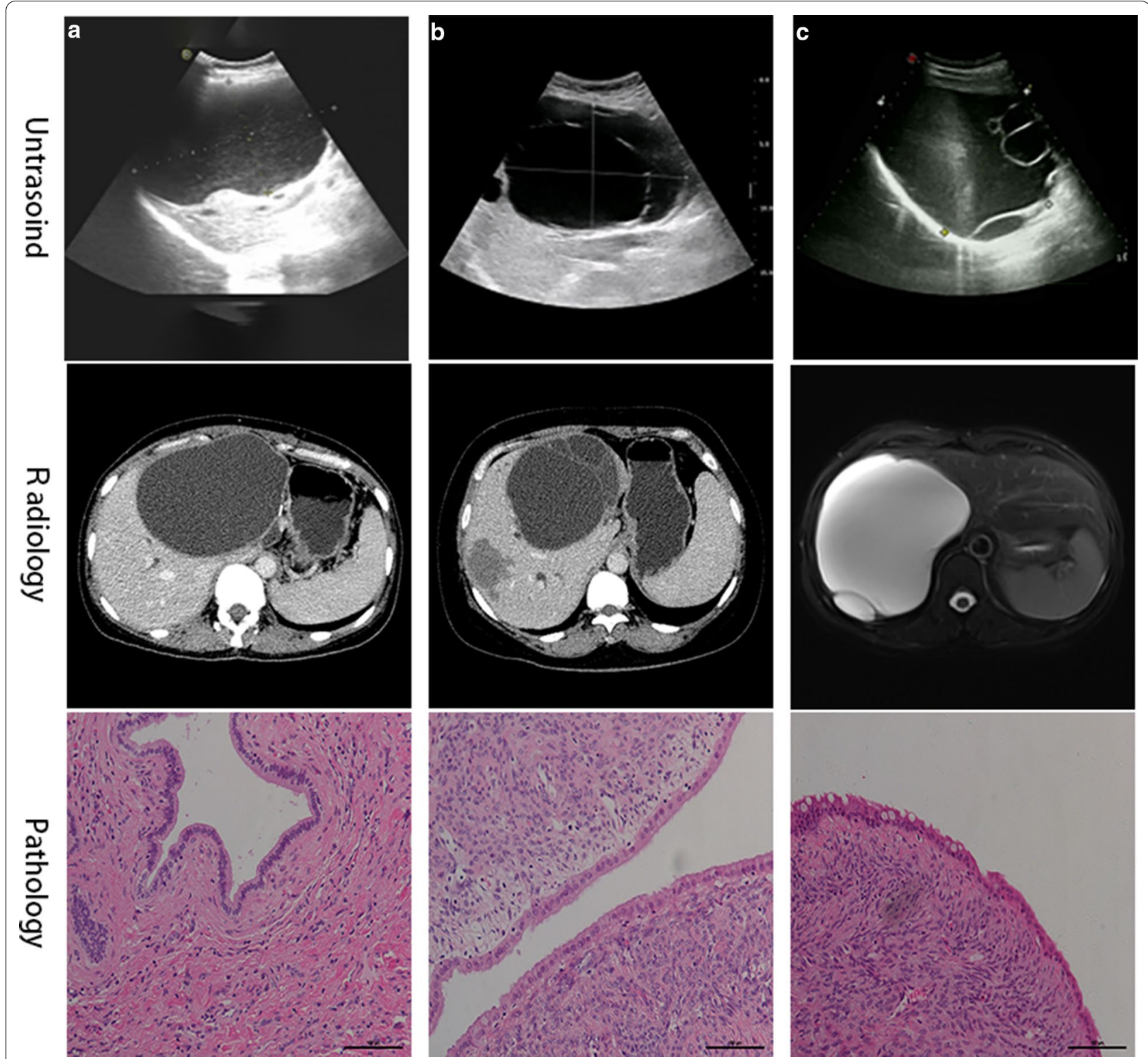

Fig. 2 Photos of contrast CT, MRI, and the pathological section of patients with hepatobiliary mucinous cystic neoplasm (H-MCNs). (Heamatoxy-lin \& eosin, $\times 40$ ) Patient A was a 40-50 years old patient who discovered the liver cystic lesion with no symptoms for 8 years. Three years ago, she underwent laparoscopic left lateral hepatic lobectomy in her local hospital. But two years later, she was found a $17 \mathrm{~cm}$ cystic tumor in the liver again. We performed the laparoscopic left hemihepatectomy and caudate lobectomy for her. Patient B was a 31-40 years old patient who discovered "hepatic simple cyst" for 10 years. Two years ago, she was diagnosed with hepatic simple cyst and underwent liver cyst fenestration in another hospital. The postoperative pathological report showed the lesion was H-MCNs but wasn't performed further treatment. She was admitted to our hospital for abdominal pain for two months. The US, CT images were showed above. We performed a laparoscopic liver cystic tumor resection and T-tube drainage for her. Patient C was a 51-60 years old patient admitted to our hospital due to being discovered a liver cystic lesion for ten years. Two years ago, she was performed fenestration, but the cyst still grew up gradually. By MRI picture, we could see a gaint cyst in the right lobe with septa. The corresponding histology (Haematoxylin and eosin staining) pictures showed OS and mucinous epithelial lining. All of those three patients were alive with no recurrence when the latest followed up

\section{Discussion}

IBC and IBAC are kinds of rare liver cystic tumours, occupying only about $5 \%$ of liver cystic lesions [4]. In 2010, IBC and IBAC were redefined as H-MCNs by
WHO new classification according to the presence of an ovarian-like stoma (OS) $[7,8]$. About $20 \%$ of BAC tends to transform into IBAC. Thus, the treatment of $\mathrm{H}-\mathrm{MCNs}$ should be more radical, and the accurate diagnosis of 
Table 1 Image examination and treatment between hepatobiliary mucinous cystic neoplasms and hepatic simple cyst

\begin{tabular}{|c|c|c|c|}
\hline & H-MCNs $(n=29)$ & $\operatorname{HSC}(n=75)$ & $p$ value \\
\hline \multicolumn{4}{|l|}{ Preoperative imaging, $\mathrm{x} / \mathrm{n}^{\dagger}(\%)$} \\
\hline Ultrasound (US) & $1 / 29(3.4)$ & $66 / 73(90.4)$ & $<0.001$ \\
\hline Contrast computed tomography $(\mathrm{CT})$ & $12 / 26(46.1)$ & $62 / 67(92.5)$ & $<0.001$ \\
\hline Magnetic resonance imaging (MRI) & $8 / 14(57.1)$ & $6 / 8(75)$ & $<0.001$ \\
\hline Operation, n (\%) & & & $<0.001$ \\
\hline Fenestration & $0(0)$ & $72(96)$ & \\
\hline Tumor resection & $10(34.5)$ & $2(2.7)$ & \\
\hline Partial liver resection & $19(65.5)$ & $1(1.3)$ & \\
\hline Surgical approach, n (\%) & & & $<0.001$ \\
\hline Laparoscopy & $14(48.3)$ & $75(100)$ & \\
\hline Open & $15(51.7)$ & $0(0)$ & \\
\hline Estimated blood lost, median (IQR) & $200(100-300)$ & $10(5-13.75)$ & $<0.001$ \\
\hline Operation time, median (IQR) & $169(213-288)$ & $55(45-90)$ & $<0.001$ \\
\hline Complication, n (\%) & $5(20)$ & $3(4)$ & 0.037 \\
\hline Abdominal bleeding & $1(3.6)$ & $0(0)$ & \\
\hline Bile leakage & $2(7.1)$ & $0(0)$ & \\
\hline Incisional infection & $1(3.6)$ & $0(0)$ & \\
\hline Pleural effusion & $0(0)$ & $1(1.3)$ & \\
\hline Abdominal collection & $0(0)$ & $1(2.6)$ & \\
\hline Incision infection & $1(3.6)$ & $0(0)$ & \\
\hline
\end{tabular}

H-MCNs hepatobiliary mucinous cystic neoplasms, HSC hepatic simple cyst

${ }^{+} \mathrm{X}$ : diagnose accurately by the equipment; $\mathrm{n}$ : the number of the patient who have done the examination

$\mathrm{H}-\mathrm{MCNs}$ and $\mathrm{HSC}$ is of great significance. However, due to the lack of specific biochemical markers and imaging features, the identification of $\mathrm{H}-\mathrm{MCNs}$ was still challenging. To improving the differential diagnosis between $\mathrm{H}-\mathrm{MCNs}$ and HSC, previous researchers make their effects on finding some specific markers. [9, 12-14]. Nevertheless, these small-sample studies can hardly find significant differences between HSC and H-MCNs. Labib et at. reported that $\mathrm{H}-\mathrm{MCNs}$ was often found as a single cyst $(p=0.006)$ [13]; Koffron et at. reported that the elevation of CA19-9 and CEA level in the cystic fluid were significantly different;[14] while Choi et al. found that cystic fluid analysis was useless to diagnose $\mathrm{H}-\mathrm{MCNs}$ [9]. At present, the imaging examination is still the most effective method to diagnose H-MCNs, although lacking reliably specific image feature [10]. Frozen section after the fenestration is also an accurate method of diagnosing $\mathrm{H}-\mathrm{MCN}$, but it would be a risk to perform such a technique if the lesion harboured a carcinoma focus. In a word, the data on the preoperative diagnosis of $\mathrm{H}-\mathrm{MCNs}$ remains scarce, and few studies were based on the new classification of H-MCNs [4-6, 8, 10, 15, 16].

In our cohort, a total of 29 patients diagnosed as $\mathrm{H}$-MCNs with the presence of OS in pathology were reviewed. Major of $\mathrm{H}-\mathrm{MCNs}$ occurred in women (20/29, $69 \%)$, particularly younger age women ( $\leq 60$ year,79\%), which is consistent with the previous study, although it didn't reach significant differences $[9,13]$. There are many studies compared the tumour markers between HSC and H-MCNs $[9,13,14,17]$. In our study, we found that serum CA19-9 level was increased in many patients with $\mathrm{H}-\mathrm{MCNs}$, but the difference was not statistically significant $(p=0.053)$. Cyst fluid tumour markers analysis of $\mathrm{H}-\mathrm{MCNs}$ remains controversial and needs more extensive sample sizes study to verify $[9,13]$. Radiographic examination was still the primary tool to diagnose H-MCNs [18]. However, we revealed that only about half of patients with $\mathrm{H}-\mathrm{MCNs}$ could be accurately diagnosed by the CT $(12 / 26,46.1 \%)$ and MRI $(8 / 14,57.1 \%)$, while the US was nearly no help to diagnose $\mathrm{H}-\mathrm{MCNs}$ $(1 / 29,3.4 \%)$. (Table 1) The imaging characteristics of $\mathrm{H}-\mathrm{MCNs}$, reported previously, were solitary, large, thickwall, multilocular cystic with internal septation and calcification [11, 18]. Nevertheless, the imaging finding of many H-MCNs were are atypical, and some HSCs may also present these features, which cause great difficulty of diagnosis.

In multivariate analysis, three factors, including biliary ductal dilation, enhancement in CT and intrahepatic location, were significantly associated with $\mathrm{H}-\mathrm{MCNs}$. $\mathrm{H}-\mathrm{MCNs}$ originate from congenitally aberrant bile ducts, and the cyst fluid is secreted by these abnormal bile duct 
Table 2 Clinical and imaging feature of the patients with the hepatic liver lesion

\begin{tabular}{|c|c|c|c|}
\hline & H-MCNs $(n=29)$ & $\operatorname{HSC}(n=75)$ & $p$-value \\
\hline \multicolumn{4}{|l|}{ Demographics } \\
\hline Age, mean(SD), year & & & 0.068 \\
\hline$>60$ & $9(31.0)$ & $40(53.3)$ & \\
\hline$\leq 60$ & $20(79.0)$ & $35(46.7)$ & \\
\hline Sex, n (\%) & & & 0.463 \\
\hline Female & $20(69.0)$ & $57(76.0)$ & \\
\hline Male & $9(31)$ & $25(24)$ & \\
\hline \multicolumn{4}{|l|}{ Symptom, n (\%) } \\
\hline Abdominal pain & $14(48.3)$ & $19(25.3)$ & 0.024 \\
\hline Abdominal fullness & $7(24.1)$ & $10(13.3)$ & 0.181 \\
\hline Fever & $4(13.8)$ & $0(0)$ & 0.005 \\
\hline Jaundice & $2(6.9)$ & $0(0)$ & 0.076 \\
\hline Weight loss & $1(3.4)$ & $0(0)$ & 0.275 \\
\hline Duration of the symptom (month) & $1(0.133-240)$ & $60(0.1-360)$ & $<0.001$ \\
\hline \multicolumn{4}{|l|}{ Liver function } \\
\hline $\mathrm{AST}(\mathrm{IQR}), \mathrm{U} / \mathrm{I}$ & $23(13-38)$ & $20(18-23)$ & 0.273 \\
\hline $\mathrm{ALT}(\mathrm{IQR}), \mathrm{U} / \mathrm{I}$ & $20(13.5-31.5)$ & $17(14-22)$ & 0.101 \\
\hline Total bilirubin (IQR), mg/dl & $13.4(11.2-16.2)$ & $12.3(10-16)$ & 0.730 \\
\hline \multicolumn{4}{|l|}{ Serum tumor markers } \\
\hline CA 19-9 (U/mL), n (\%) & & & 0.001 \\
\hline$>20$ & $18(64.3)$ & $15(27.3)$ & \\
\hline$\leq 20$ & $10(35.7)$ & $40(72.7)$ & \\
\hline CEA (ng/mL), n (\%) & & & 0.469 \\
\hline$>5$ & $2(7.1)$ & $2(2.7)$ & \\
\hline$\leq 5$ & $26(92.9)$ & $54(72.0)$ & \\
\hline CA 12-5 (U/mL), n (\%) & & & 0.117 \\
\hline$>20$ & $6(20.7)$ & $5(9.1)$ & \\
\hline$\leq 20$ & $22(75.9)$ & $50(90.9)$ & \\
\hline $\operatorname{AFP}(\mathrm{ng} / \mathrm{ml}), \mathrm{n}(\%)$ & & & 0.598 \\
\hline$>10$ & $2(7.1)$ & $2(3.6)$ & \\
\hline$\leq 10$ & $26(92.9)$ & $54(96.4)$ & \\
\hline \multicolumn{4}{|l|}{ Imaging feature ${ }^{\dagger}, \mathrm{n}(\%)$} \\
\hline Biliary ductal dilation & 17 (58.6) & $4(5.3)$ & $<0.001$ \\
\hline Multilocular cyst & $17(58.6)$ & $26(83.9)$ & 0.109 \\
\hline Enhancement after contrast & $20(69.0)$ & $11(14.7)$ & $<0.001$ \\
\hline Septa & $13(44.8)$ & $12(16.0)$ & 0.002 \\
\hline Calcification & $2(6.9)$ & $12(16.0)$ & 0.223 \\
\hline Cyst size (cm), median (IQR) & $8.5(3.7-12.3)$ & $9.1(7.8-11.0)$ & 0.131 \\
\hline Location of the cyst, $\mathrm{n}(\%)$ & & & $<0.001$ \\
\hline Left & $23(79.3)$ & $22(29.2)$ & \\
\hline Right & $3(10.3)$ & $39(58)$ & \\
\hline Both & $3(10.3)$ & $14(18.7)$ & \\
\hline
\end{tabular}

${ }^{\dagger}$ Imaging features were collected based on abdominal contrast CT or abdominal contrast MRI

cells. Compared with HSC, there is more blood supply in the wall, nodules and septa of H-MCNs. AS a result, the $\mathrm{H}$-MCNs was often combined with bile duct dilatation and enhanced after contrast in imaging. What's interesting was that although the normal right lobe of the liver is much bigger than the left, most lesions of $\mathrm{H}-\mathrm{MCNs}$ $(23 / 29,79.3 \%)$ located in the left lobe and was statistically significant in multivariate analysis. Zen et al. and Albores-Saavedra et al. found a similar phenomenon [19, 20]. In our view, this may because the high degree of left 
Table 3 Multivariate statistical analyses of Clinical characteristics in HSC and H-MCNs

\begin{tabular}{lll}
\hline & OR $(95 \% \mathrm{Cl})$ & p-value \\
\hline Abdominal pain & $3.71(0.572-29.8)$ & 0.178 \\
Fever & NA & 0.995 \\
Duration of the symptom & $0.97(0.985-1.00)$ & 0.600 \\
CA199 & $5.21(1.02-31.9)$ & 0.053 \\
Enhancement after contrast & $12.1(2.11-100)$ & $0.009^{* *}$ \\
Intrahepatic location & & \\
Right & - & - \\
Left & $11.0(1.71-121)$ & $0.020^{*}$ \\
Both & $7.56(0.871-108)$ & 0.087 \\
Biliary ductal dilation & $9.80(1.46-92.2)$ & $0.027^{* *}$ \\
Septa & $2.89(0.455-20.3)$ & 0.260 \\
\hline
\end{tabular}

OR odds radio, $\mathrm{Cl}$ confidence interval

liver freeness, the tumour is prong to outward compression growth in the loose tissue, the mechanism need to be further explored.

The preoperative distinguish of $\mathrm{H}-\mathrm{MCNs}$ and $\mathrm{HSC}$ was crucial to make the surgical plan. Because of the high rate of recurrence and premalignant progression, $\mathrm{H}-\mathrm{MCNs}$ were recommended to undergo radical resection, such as complete cyst resection or anatomical liver resection, even liver transplantation [10, 21, 22]. It was reported that complete cyst resection could bring to long-term survival and a low rate of recurrence [10,23]. According to a multi-institutional study, if the $\mathrm{H}$-MCNs were performed fenestration, the recurrence could reach $48.6 \%$, and might enhance the risk of malignant-transformation [10]. The treatment of large HSC is usually laparoscopic fenestration or unroofing when patients have the symptom of compression [25]. As a result, several HSC misdiagnosed as H-MCNs may undergo radical excision to suffer unnecessary surgical trauma.

Since the preoperative diagnosis of $\mathrm{H}-\mathrm{MCNs}$ remains quite tricky, it is essential to establish a preoperative diagnosis model to enhance the differential ability of $\mathrm{H}-\mathrm{MCNs}$ and HSC. We created a diagnosis nomogram that facilitates accurate identification and screening of $\mathrm{H}$-MCNs preoperatively. Nomogram is a visualized tool that could vividly display the logistic regression model and help to make clinical decisions. In our Nomogram, three factors (Enhancement after contrast, intrahepatic

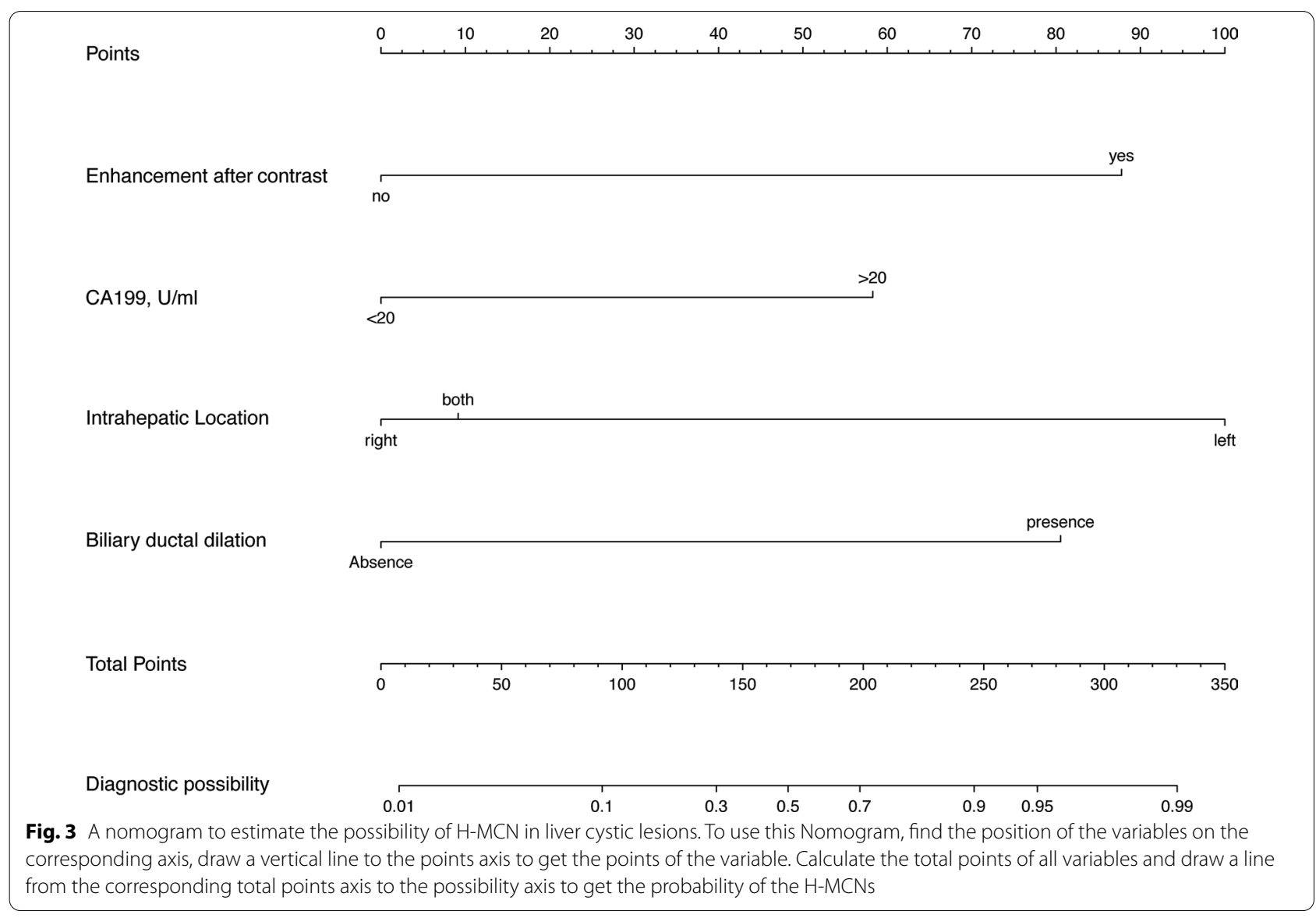




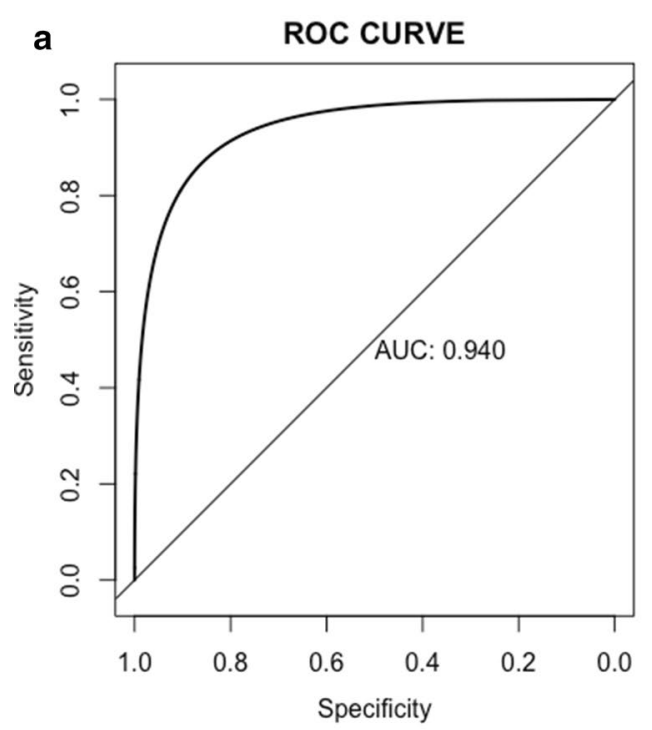

b

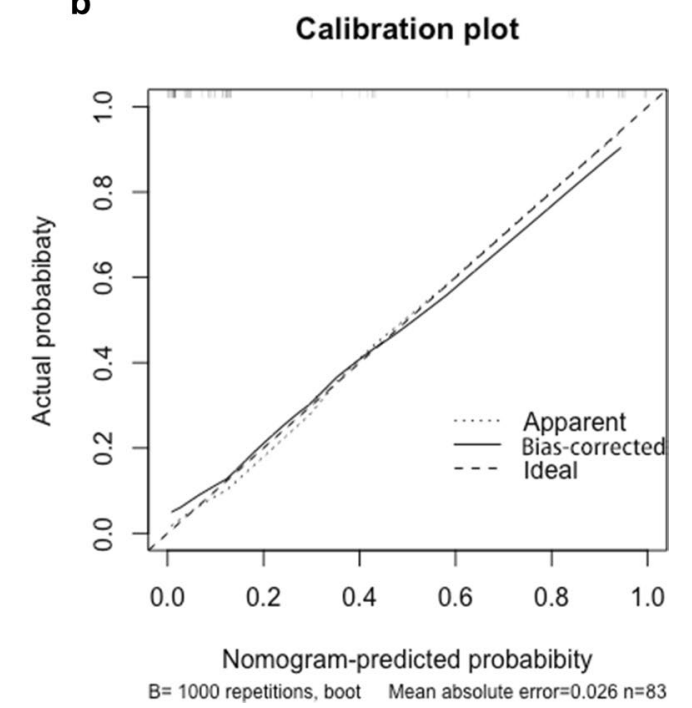

Fig. 4 a ROC curve of the H-MCNs prediction nomogram. The ROC curve of the model showed excellent discrimination (C-index $=0.940$ ). $\mathbf{b}$ Calibration plot for predicting the probability of H-MCNs in the hepatic cystic lesion. The predicted probability of the Nomogram was good agreement with the actual probability

location, and biliary dilation) were significantly associated with $\mathrm{H}-\mathrm{MCNs}$ in the multivariate logistic regression analysis were included. Besides, serum CA19-9 level was widely reported to associate with $\mathrm{H}-\mathrm{MCNs}$ in previous studies $[10,24,25]$. In our analysis, serum CA19-9 level was increased in many patients with $\mathrm{H}-\mathrm{MCNs}$, but the difference was not statistically significant in multivariate analysis $(p=0.053)$. In consideration of improving the sensitivity of the diagnostic model, we also included the CA19-9 as a significant factor. This is the first Nomogram of $\mathrm{H}-\mathrm{MCNs}$ in liver cystic lesions. The advantages of the Nomogram were that it provides individualized risk assessment in a dynamic manner, and all the factors included are routinely reached in clinical practice. By utilizing this Nomogram, the patients with a high probability of H-MCNs would be recommended to undergo a complete resection. And some preoperatively invasive tests like biopsy or fluid aspiration would be replaced by using the Nomogram to get a risk probability.

There were some limitations to this study. First of all, the current study was a retrospective study with some inevitable selection bias. Second, although the H-MCNs were a rare disease, the sample size of the present study was too small and we have no ability to include enough samples for impeccable logistic regression. Thus, the Nomogram based on these 29 positive cases may not fully reflect the reality, and large-sample multicenter studies were still expected in the future. Before the wide acceptance of this Nomogram, it is desirable to validate this result from other centres. Third, considering whether the cystic fluid tumour marker level could help in the diagnosis of $\mathrm{H}-\mathrm{MCNs}$ remains controversial, and not all of the liver cystic patients were routinely performed aspiration paracentesis, we didn't include fluid tumour marker level as a predictive factor. Meanwhile, other specific markers understudy might further improve the accuracy of the prediction model of H-MCNs.

\section{Conclusion}

$\mathrm{H}-\mathrm{MCN}$ sere rare cystic tumour of the liver, which are difficulty in diagnosis. Enhancement after contrast, located in the left lobe of the liver and biliary ductal dilation, are significantly independent predictors to distinguish between $\mathrm{H}-\mathrm{MCNs}$ and $\mathrm{HSC}$. The $\mathrm{H}-\mathrm{MCNs}$ risk predictive nomogram provides an accurate probability of $\mathrm{H}-\mathrm{MCNs}$ risk in liver cystic lesions and helps to make the clinical decision.

\section{Abbreviations}

H-MCNs: Hepatobiliary mucinous cystic neoplasms; HSC: Hepatic simple cyst; IBC: Intrahepatic biliary cystadenomas; IBAC: Intrahepatic biliary cystadenocarcinoma; OS: Ovarian-like stroma; WHO: World Health Organization; US: Ultrasound; CT: Computed tomography; MRI: Magnetic resonance imaging; C-index: Concordance index.

\section{Supplementary Information}

The online version contains supplementary material available at https://doi. org/10.1186/s12893-021-01110-9.

Additional file 1: Table. Characteristic and clinical feature of the patients with hepatobiliary mucinous cystic neoplasm (H-MCNs). 


\section{Acknowledgements}

Thanks to the patients who participated in the study.

\section{Authors' contributions}

X. L and JQ. G: Study/Protocol design; JH. Z, JW.C, M. A. K: Data collection and management; JW. C, JJ. X, LY.T, J. F: Data analysis; JH. Z: Writing the manuscript. JQ. G, YL. L, X. F, JJ. X, M. A. K: Reviewing and revising the manuscript. All authors have read and approved the final manuscript.

\section{Funding}

This study was supported by the National Natural Science Foundation of China (Grant No. 82072625) and the Zhejiang Major Medical Science and Technology Plan supported by National Health Commission of China (Grant No. WKJ-ZJ-2030). The funding bodys had no influence on the design of the study and collection, analysis, and interpretation of data and in writing the manuscript.

\section{Availability of data and materials}

We declared that materials described in the manuscript, including all relevant raw data, will be freely available to any scientist wishing to use them for noncommercial purposes, without breaching participant confidentiality. The data is available from the corresponding author.

\section{Ethics approval and consent to participate}

This study was approved by the Ethics committee of Sir Run Run Shaw Hospital (Reference number SRR20200210). All of the participants signed the consent of the study. Informed consent to participate in the study were obtained from participants.

\section{Consent for publication}

Not applicable.

\section{Competing interests}

All authors have none competing interest to declare.

Received: 20 April 2020 Accepted: 21 February 2021

Published online: 20 March 2021

\section{References}

1. Carrim ZI, Murchison JT. The prevalence of simple renal and hepatic cysts detected by spiral computed tomography. Clin Radiol. 2003;58(8):626-9.

2. Fiamingo $P$, et al. Laparoscopic treatment of simple hepatic cysts and polycystic liver disease. Surg Endosc. 2003;17(4):623-6.

3. Walt AJ. Cysts and benign tumors of the liver. Surg Clin North Am. 1977;57(2):449-64.

4. Vogt DP, Henderson JM, Chmielewski E. Cystadenoma and cystadenocarcinoma of the liver: a single center experience. J Am Coll Surg. 2005;200(5):727-33.

5. Henson Jr. SW, Gray HK, Dockerty MB. Benign tumors of the liver. VI. Multilocular cystadenomas. Surg Gynecol Obstet. 1957;104(5):551-4.

6. Devaney K, Goodman ZD, Ishak KG. Hepatobiliary cystadenoma and cystadenocarcinoma. A light microscopic and immunohistochemical study of 70 patients. Am J Surg Pathol. 1994;18(11):1078-91.

7. Bosman FT, Carneiro F, Hruban RH, Theise ND. WHO classification of tumors of the digestive system. 4th ed. Lyon: International Agency for Research on Cancer Publisher; 2010.
8. Quigley B, et al. Hepatobiliary mucinous cystic neoplasms with ovarian type stroma (so-called "hepatobiliary cystadenoma/cystadenocarcinoma"): clinicopathologic analysis of 36 cases illustrates rarity of carcinomatous change. Am J Surg Pathol. 2018;42(1):95-102.

9. Choi HK, et al. Differential diagnosis for intrahepatic biliary cystadenoma and hepatic simple cyst: significance of cystic fluid analysis and radiologic findings. J Clin Gastroenterol. 2010;44(4):289-93.

10. Arnaoutakis DJ, et al. Management of biliary cystic tumors: a multi-institutional analysis of a rare liver tumor. Ann Surg. 2015;261(2):361-7.

11. Wang $C$, et al. Intrahepatic biliary cystadenoma and cystadenocarcinoma: an experience of 30 cases. Dig Liver Dis. 2012;44(5):426-31.

12. Lantinga MA, Gevers TJ, Drenth JP. Evaluation of hepatic cystic lesions. World J Gastroenterol. 2013;19(23):3543-54.

13. Labib PLZ, et al. Differentiating simple hepatic cysts from mucinous cystic neoplasms: radiological features, cyst fluid tumour marker analysis and multidisciplinary team outcomes. Dig Surg. 2017;34(1):36-42.

14. Koffron A, et al. Intrahepatic biliary cystadenoma: role of cyst fluid analysis and surgical management in the laparoscopic era. Surgery. 2004;136(4):926-36

15. Fairchild R, et al. Biliary cystadenoma: a case report and review of the literature. Mo Med. 1993;90(10):656-7.

16. Wang K, et al. Diagnosis and treatment of intrahepatic biliary cystadenoma: experience with 14 cases in a single center. Med Oncol. 2014;31(11):274.

17. Fuks $D$, et al. Intracystic concentrations of tumour markers for the diagnosis of cystic liver lesions. Br J Surg. 2014;101(4):408-16.

18. Pojchamarnwiputh $\mathrm{S}$, et al. Computed tomography of biliary cystadenoma and biliary cystadenocarcinoma. Singapore Med J. 2008:49(5):392-6.

19. Zen Y, et al. Intraductal papillary neoplasms and mucinous cystic neoplasms of the hepatobiliary system: demographic differences between Asian and Western populations, and comparison with pancreatic counterparts. Histopathology. 2014;65(2):164-73.

20. Albores-Saavedra J, et al. Cystadenomas of the liver and extrahepatic bile ducts: morphologic and immunohistochemical characterization of the biliary and intestinal variants. Ann Diagn Pathol. 2015;19(3):124-9.

21. Li H, Peng B. Laparoscopic anatomical left hepatectomy for intrahepatic bile duct papillary mucinous cystadenoma with intraoperative vascular repair: a case report. Medicine (Baltimore). 2016;95(6):e2802.

22. Chen YW, et al. Surgical management of biliary cystadenoma and cystadenocarcinoma of the liver. Genet Mol Res. 2014;13(3):6383-90.

23. Soares KC, et al. Cystic neoplasms of the liver: biliary cystadenoma and cystadenocarcinoma. J Am Coll Surg. 2014;218(1):119-28.

24. Zhang FB, et al. Preoperative differential diagnosis between intrahepatic biliary cystadenoma and cystadenocarcinoma: a single-center experience. World J Gastroenterol. 2014;20(35):12595-601.

25. Sumiyoshi T, et al. Mucinous cholangiocarcinoma: clinicopathological features of the rarest type of cholangiocarcinoma. Ann Gastroenterol Surg. 2017;1(2):114-21.

\section{Publisher's Note}

Springer Nature remains neutral with regard to jurisdictional claims in published maps and institutional affiliations. 